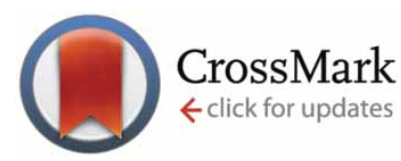

\title{
Peanut allergy: how common is it and why?
}

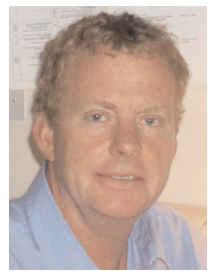

\section{P. Cullinan}

Dept of Occupational and Environmental Medicine Imperial College School of Medicine

London

SW3 6LR

UK

Fax: 442073518336

E-mail:p.cullinan@ imperial.ac.uk
Despite its (English) name, the peanut is a bean rather than a nut; it is botanically distinct from "tree nuts" such as walnuts, hazelnuts and almonds. Like other "nuts", the peanut is capable, on ingestion, of inducing an allergic response - indeed peanut allergy is notorious for its often serious, and sometimes fatal, anaphylactic nature. This, the frequency with which it occurs in children and concerns that it may be an increasingly common problem have together contributed to widespread professional interest in, and public fear over, peanut allergy.

As with most diseases, important clues to the aetiology of peanut allergy are likely to be found in its basic, descriptive epidemiology. Unfortunately we have limited understanding of apparently simple issues such as: how common is peanut allergy? What is its distribution by age? And how is it related to time and place? In part, this reflects real difficulties in making populationbased measurements of food allergies. Selfreported disease is often, in perhaps 10\% of cases, not accompanied by evidence of specific immunoglobulin (Ig)E sensitisation or by a positive response to blinded challenge. Further difficulties arise from making epidemiological - and aetiological - inferences from the high proportion of publications derived from clinical case series.

Among European children, the prevalence of specific IgE sensitisation to peanut allergens has been estimated to be about 3\% [1]; approximately half of these children reported an associated allergic response. Similar prevalences have been reported in North American children $[2,3]$. Each of these important surveys suffered from a relatively low response rate. In addition, probable responder bias and the increasing public awareness of peanut allergy mean that their estimates may be on the high side. There is even less highquality information for adults but self-reports suggest similar prevalences to those found in children $[3,4]$. Peanut allergy is consistently associated with atopy and is almost always accompanied by other allergic disease. As with other atopic conditions, it is more common among male children, although the sex difference diminishes and may even be reversed in adulthood. There is, as yet, very little reliable information on its geographical distribution, but what is available suggests that it reflects that of other allergies, with a higher frequency in more economically advantaged countries and, apparently, comparative rarity in sub-Saharan Africa and parts of Asia [5]. Better information should soon be available from the international EuroPrevall surveys, which are due to report next year.

But it is the issue of whether peanut allergy is more common than it used to be that has excited most attention. Few allergists - and fewer journalists - would doubt this, but a close look at the available evidence is less compelling. Probably the strongest evidence is derived from the Isle of Wight (UK), where two successive cohorts of children - the first born in 1989 and the second between 1994 and 1996 - were surveyed at the age of 3-4 years [1]. Questionnaires were accompanied by skin-prick testing with peanut allergens and, where indicated, by oral challenge testing. In the second survey, just 44\% of children underwent skin testing. Specific sensitisation to peanut was three times as common $(3.3 \%)$ in the second cohort, a difference that was statistically significant $(p=0.001)$. Reported allergy to peanut $(1.0 \%)$ was also more common, by a factor of two, although this difference may readily have arisen by chance $(p=0.17)$. Peanut sensitisation in the second cohort was strongly associated with asthma, hayfever and particularly with a history of eczema. The other widely quoted study is a telephone-based survey (53\% response rate) of adults and children in the USA, carried out in 2002 [3]. In comparison with a similar survey made 5 years earlier, a 
doubling (to $0.8 \%$ ) of the prevalence of reported peanut allergy among children aged $<18$ years was estimated. There was no difference among adults. This survey did not use objective tests.

Thus it is probably, but not certainly, the case that sensitisation (and clinical allergy) to peanuts is more common among children in some countries than it used to be. Whether this is also true among adults is unknown, but the available evidence suggests that peanut allergy rarely remits [6] and any increase in childhood incidence is likely to be transmitted to future adult generations. A temporal increase in peanut allergy is likely to be a manifestation of the general rise in other childhood allergies and a reflection of the availability of peanut as an allergen. Peanut consumption has increased in European children (and their mothers) over the past 50 years in line with many other food imports from the USA. Intriguingly, in countries where there is heavy consumption of boiled (rather than roasted) peanuts, allergy appears to be very uncommon. The observation that allergic reactions in young children are commonly evident on what appears to be the first (oral) exposure has raised the question of how and when initial sensitisation takes place, with particular interest in women's diets during pregnancy and while breastfeeding. On the basis of remarkably little evidence, a precautionary approach, whereby prospective mothers and those with infant children are advised to avoid all peanut consumption, has been widely taken. Finally, there has been considerable interest in other routes of exposure, prompted by the publication of an elegant epidemiological study from the UK demonstrating a strong association between

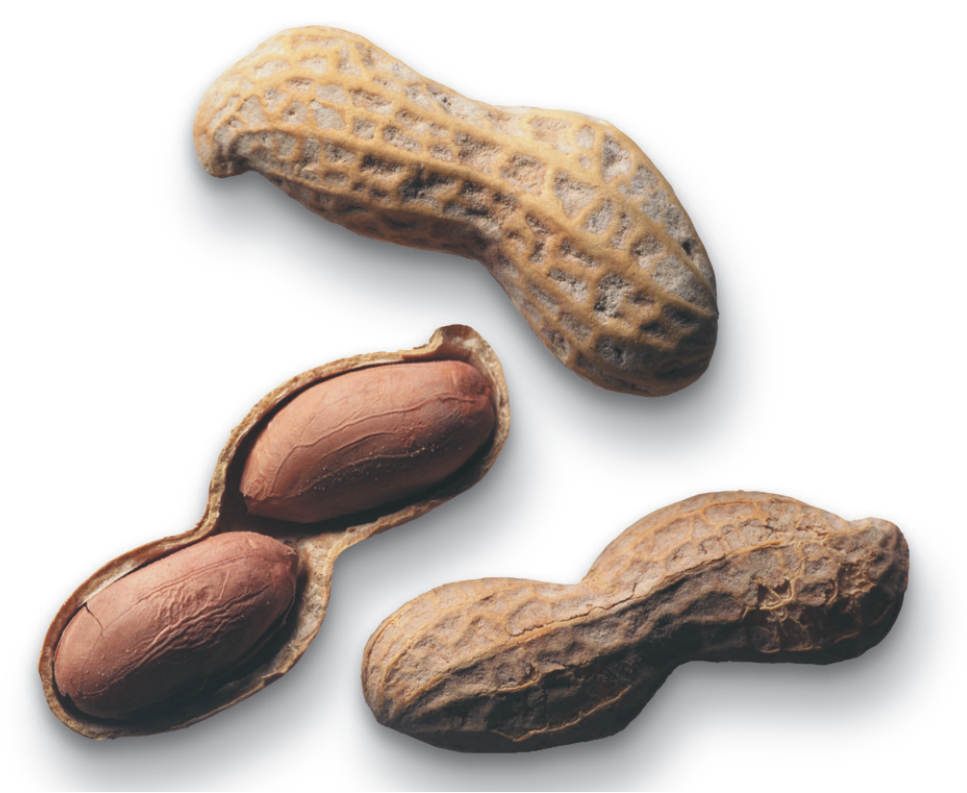

peanut allergy and the use of skin creams containing peanut oil [7]. In most cases, the creams were probably used for the treatment or prevention of common nappy rash, but there was also an independent relationship with eczematous skin damage. It remains unclear how much of the population burden of peanut allergy in different countries might be attributable to sensitisation acquired through the skin in this way.

While (roasted) peanuts are eaten and while peanut oils are widely used, peanut allergy will not disappear. Indeed the problem is likely to become more common as we inherit an increasingly atopic population. Until and unless more is learned about the specific factors that induce peanut sensitisation, its management will have to rely on avoidance, adrenaline and increasingly sophisticated anti-allergy treatments.

\section{References}

1. Grundy J, Matthews S, Bateman B, Dean T, Arshad SH. Rising prevalence of allergy to peanut in children: data from 2 sequential cohorts. J Allergy Clin Immunol 2002; 110: 784-789.

2. Kagan RS, Joseph L, Dufresne C, et al. Prevalence of peanut allergy in primary-school children in Montreal, Canada. J Allergy Clin Immunol 2003; 112: 1223-1228.

3. Sicherer SH, Munoz-Furlong A, Sampson HA. Prevalence of peanut and tree nut allergy in the United States determined by means of a random digit dial telephone survey: a 5-year follow-up study. J Allergy Clin Immunol 2003; 112: 1203-1207.

4. Emmett SE, Angus FJ, Fry JS, Lee PN. Perceived prevalence of peanut allergy in Great Britain and its association with other atopic conditions and with peanut allergy in other household members. Allergy 1999; 54: 380-385.

5. Hill DJ, Hosking CS, Heine RG. Clinical spectrum of food allergy in children in Australia and South-East Asia: identification and targets for treatment. Ann Med 1999; 31: 272-281.

6. Vander Leek TK, Liu AH, Stefanski K, Blacker B, Bock SA. The natural history of peanut allergy in young children and its association with serum peanut-specific IgE. J Pediatr 2000; 137: 749-755.

7. Lack G, Fox D, Northstone K, Golding J. Factors associated with the development of peanut allergy in childhood. N Engl J Med 2003; 348: 977-985. 